\title{
Identification of hysteretic systems with slip using bootstrap filter
}

\author{
S.J. Li ${ }^{\text {a,* }}$, Y. Suzuki ${ }^{b}$, M. Noori ${ }^{\mathrm{c}}$ \\ anstitute of Civil Engineering and Architecture, Wuhan University of Technology, 122 Luo Shi Road, \\ Wuhan, Hubei 430070, People's Republic of China \\ ${ }^{\mathrm{b}}$ Disaster Prevention Research Institute, Kyoto University, Uji, Kyoto 611, Japan \\ ${ }^{\mathrm{c}}$ Department of Mechanical and Aerospace Engineering, North Carolina State University, NC 27695-7910, USA
}

\begin{abstract}
Hysteretic models with slip are frequently used to predict the non-linear behaviour of many structural systems, for example wood buildings and reinforced concrete structures. A model, called SL model, which can describe the pinching of most practical hysteresis loops perfectly was proposed by Baber and Noori. This model is characterised by control parameters that have to be identified from observed experimental data. A method of estimating the parameters of SL model on the basis of input-output data based on Bayesian state estimation and bootstrap filter is suggested in this paper, which has the great advantages of being able to handle any functional non-linearity and system and measurement noise of any distribution. A numerical simulation shows its suitability and effective for the system even in the case of very severe material non-linearity.
\end{abstract}

\section{Introduction}

Many civil engineering structures exhibit hysteresis when subjected to severe dynamic loading, i.e., the restoring force of structure depends not only on the instantaneous deformation but also on the past history of deformation. As a result, the hysteretic restoring force cannot be expressed by an algebraic function of the instantaneous displacement and velocity.

\footnotetext{
*Corresponding author.

E-mail addresses: sj-li@peoplemail.com.cn (S.J. Li), suzuki@zeisei.dpri.kyoto-u.ac.jp (Y. Suzuki), mohammad_noori@ncsu.edu (M. Noori).
} 
This memory nature renders the hysteretic systems more difficult to model and analyse than other non-linear systems, especially for the hysteresis with slip. The modelling and identification of these hysteretic systems is a problem of considerable theoretical and practical interest over the years because of its importance in response prediction, structural control and health monitoring.

The class of hysteretic systems is typical of non-linear systems which involve non-linearity both in damping and stiffness. Much effort has been devoted by numerous investigators to develop models of hysteretic systems and a few models were proposed [1]. One of the widely accepted model is a differential model originally proposed by Bouc [2] and further developed and generalised by Wen and his colleagues [3,4]. In this model, the restoring force and the deformation are connected through a non-linear differential equation containing unspecified parameters. By choosing the parameters suitably, it is possible to generate a large variety of different shapes of the hysteresis loops. Based on the Bouc-Wen model, a smoothed hysteretic slip model, which can describe the pinching of hysteresis loops perfectly was proposed by Baber and Noori [5]. The model, called SL model, consists of a non-pinching hysteretic element in series with a 'slip-lock' element and contains six loop parameters. Here the parameter identification of this model is to be considered.

Identification is the process of developing an accurate mathematical model for a system, given a set of inputs and corresponding output measurements. As an on-line identification method, extended Kalman filter has received much attention and has been used successfully in the parameter estimation problems over the past years by regarding each of the parameters involved in the system as an augmented state variable [6,7]. There the estimation problem is linearised about the predicted state so that the Kalman filter can be applied and the required PDF is still approximated by a Gaussian, which may be a gross distortion of the true underlying structure and may lead to estimation divergence.

A filtering method, called bootstrap filter, based on Bayesian state estimation and Monte-Carlo method was proposed by Gordon [8], which has the great advantage of being able to handle any functional non-linearity and system and/or measurement noise of any distribution. Another similar method is the Monte-Carlo filter proposed by Kitagawa [9]. The central idea of these methods is to represent the required PDF as a set of random samples, rather than as a function over state space to avoid the limitations mentioned above. As the number of samples becomes very large, they can effectively provide an exact, equivalent, representation of the required PDF. Estimations of states can be obtained directly from the samples. Using these filtering techniques, methods for identification of parameters of non-linear and non-Gaussian models subjected to dynamic forces were discussed by Hoshiya and others [10-13].

In the past decades, efforts have been devoted to developing the identification procedures for non-linear hysteretic systems [14-17]. But most of them were presented by referring to the BoucWen model and other linear hysteretic models due to their significant advantages. There is seldom paper on the estimation of hysteretic systems with slip because of its complexity.

In this paper, a parametric identification method for the SL model based on bootstrap filter is developed. And considering the fact that the SL model is complex and the number of parameters in it is large, a method to decide the initial estimates of parameters of this model is suggested to obtain stable solutions as well as their fast convergency to the optimal values. 


\section{Hysteretic slip model}

The differential system model proposed by Baber and Noori [5] to describe hysteretical system with slip is

$$
\begin{aligned}
& \ddot{x}+2 \zeta \omega_{0} \dot{x}+\alpha \omega_{0}^{2} x+(1-\alpha) \omega_{0}^{2} \bar{z}=p(t), \\
& \dot{\bar{z}}=\bar{A} \dot{x}_{1}-\bar{\beta}\left|\dot{x}_{1}\right| \bar{z}|\bar{z}|^{n-1}-\bar{\gamma} \dot{x}_{1}|\bar{z}|^{n}, \\
& \dot{x}_{2}=f(\bar{z}) \dot{\bar{z}} \\
& x=x_{1}+x_{2},
\end{aligned}
$$

in which $p(t)=P(t) / m$ is the input, $\alpha$ is the ratio of post-yield/pre-yield stiffness and $\bar{z}$ is the hysteretic restoring force. The overdot denotes the differentiation with respect to time. $\bar{A}, \bar{\beta}, \bar{\gamma}$ and $n$ are model parameters, in which $\bar{\beta}, \bar{\gamma}$ and $n$ determine the hysteresis shape, and $\bar{A}$ determines the tangent stiffness. Hysteresis loop pinching is added by incorporating a time-dependent 'slip-lock' element as shown in Figs. 1 and 2, and the relationship between the slip-lock and hysteretic restoring force is described by Eqs. (3) and (4) in rate equation form. Here the function $f(\bar{z})$ has the following properties:

(1) $f(\bar{z})$ is an, at least, piecewise continuous function, independent of the sign of $\bar{z}$ and $x_{2}$;

(2) $f(\bar{z})$ is zero, or nearly zero everywhere, except within a small region near $\bar{z}=0$, where it has a sharp peak. In the limit, as the slope during slipping goes to zero, $f(\bar{z})$ approaches the delta function. In practical situations, a large but finite peak value is expected.

For practical modelling purposes, it is convenient to choose $f(\bar{z})$ of the form

$$
f(\bar{z})=2 s g(\bar{z}),
$$

in which $s$ is the magnitude of slip and $2 s$ is the length of the slip zone. $g(\bar{z})$ is chosen to have an area of 1 , in order to allow a total slip of $2 s$. Thus, it is seen that $g(\bar{z})$ has the form of a unimodal probability density function, symmetric about $\bar{z}=0$. Although any function that has the desired attributes is suitable for $g(\bar{z})$, the Gaussian density function, with a small value of $\sigma$ to create a

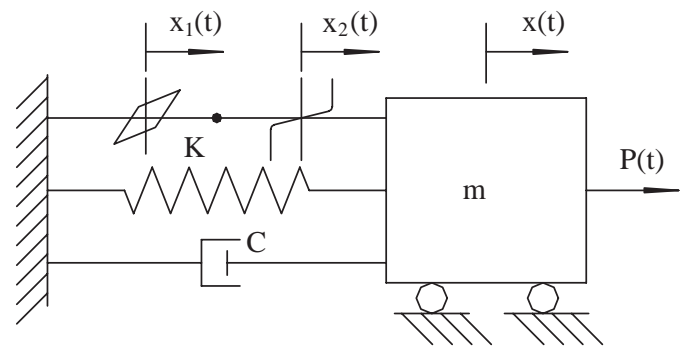

Fig. 1. System model of SDOF. 


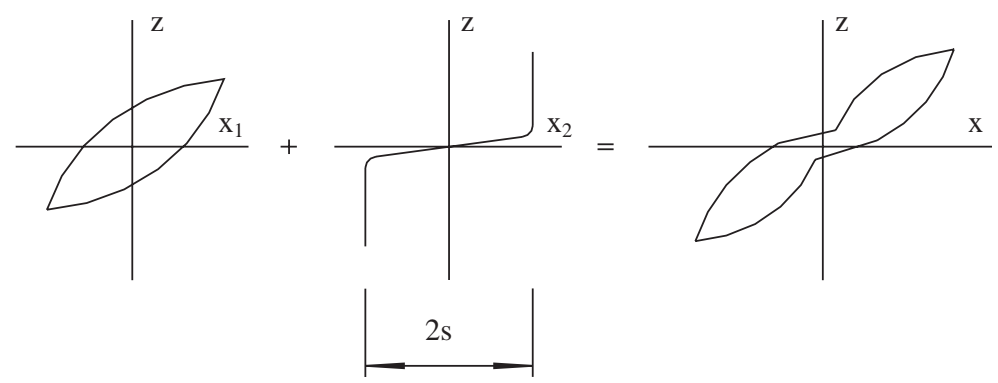

Fig. 2. Slip-lock series hysteresis.

sharp peak, has been selected for mathematical tractability. Thus,

$$
\dot{x}_{2}=\sqrt{\frac{2}{\pi}} \frac{s}{\sigma} \exp \left[-\frac{\bar{z}^{2}}{2 \sigma^{2}}\right] \dot{\bar{z}}
$$

will give a slip of $2 s$ when $\bar{z}$ changes sign.

By means of the transformation

$$
\begin{aligned}
& a=2 \zeta \omega_{0}, \quad b=\alpha \omega_{0}^{2}, \\
& z=(1-\alpha) \omega_{0}^{2} \bar{z}, \quad A=(1-\alpha) \omega_{0}^{2} \bar{A}, \\
& \beta=\bar{\beta} /\left[(1-\alpha) \omega_{0}^{2}\right]^{n-1}, \quad \gamma=\bar{\gamma} /\left[(1-\alpha) \omega_{0}^{2}\right]^{n-1},
\end{aligned}
$$

the differential equations of system can be rewritten as

$$
\begin{aligned}
& \ddot{x}+a \dot{x}+b x+z=p(t), \\
& \dot{z}=A \dot{x}_{1}-\beta\left|\dot{x}_{1}\right| z|z|^{n-1}-\gamma \dot{x}_{1}|z|^{n}, \\
& \dot{x}_{2}=\sqrt{\frac{2}{\pi}} \frac{s}{\sigma} \exp \left[-\frac{z^{2}}{2 \sigma^{2}}\right] \dot{z}, \\
& x=x_{1}+x_{2} .
\end{aligned}
$$

In general, the magnitude of slip $s$ may be varied with the history of response. In this model, $s$ is supposed as a function of the system energy dissipation $E(t)$ as follows:

$$
s=\delta_{s} E(t),
$$

where $\delta_{s}$ is a constant specified for the rate of varying of slip.

Eqs. (7)-(11) complete the model, and the parameters need to be identified are $a, b, A, \beta, \gamma, n, \delta_{s}$ and $\sigma$ for this model.

\section{Bootstrap filter}

Prior to discussing the identification of hysteretic system with slip, a summary of the bootstrap filter is made. 


\subsection{Bayesian recursion}

The discrete time estimation problem is discussed here. The state vector, $x_{k} \in R^{n}$ is assumed to evolve according to the following system model:

$$
x_{k}=f\left(x_{k-1}, w_{k}\right),
$$

where $f: R^{n} \times R^{m} \rightarrow R^{n}$ is the system transition function and $w_{k} \in R^{m}$ is zero-mean process noise independent of past and current states. The PDF of $w_{k}$ is assumed to be known as $p_{w}\left(w_{k}\right)$. At discrete times, measurements $y_{k} \in R^{p}$ become available. These measurements are related to the state vector via the observation equation

$$
y_{k}=h\left(x_{k}\right)+v_{k},
$$

where $h \in R^{p}$ is the measurement function and $v_{k} \in R^{p}$ is the observation noise, assumed to be another zero-mean random sequence independent of past and present states and the system noise $w_{k}$. The PDF of $v_{k}$ is assumed to be known as $p_{v}\left(v_{k}\right)$. Suffix $k$ stands for a discrete time in sequential order. The available information at time step $k$ is the set of measurements $D_{k}=\left\{y_{i}\right.$ : $i=1, \ldots, k\}$. It is noted that $f\left(x_{k-1}, w_{k}\right)$ and $h\left(x_{k}\right)$ are generally not linear functions and $x_{k}, w_{k}$ and $v_{k}$ are not necessarily Gaussian vectors.

The requirement is to construct the PDF of the current state $x_{k}$, given all the available information: $p\left(x_{k} \mid D_{k}\right)$. In principle, this PDF may be obtained recursively in two stages: prediction and update. Suppose that the required PDF $p\left(x_{k-1} \mid D_{k-1}\right)$ at time step $k-1$ is available. Then using the system model it is possible to obtain the prior PDF of the state at time step $k$

$$
p\left(x_{k} \mid D_{k-1}\right)=\int p\left(x_{k} \mid x_{k-1}\right) p\left(x_{k-1} \mid D_{k-1}\right) \mathrm{d} x_{k-1},
$$

where $p\left(x_{k} \mid x_{k-1}\right)$ is determined by $f\left(x_{k-1}, w_{k}\right)$ and the distribution of $w_{k}$ in the system equation (12). Then at time step $k$ a measurement $y_{k}$ becomes available and may be used to update the prior through Bayes rule

$$
p\left(x_{k} \mid D_{k}\right)=\frac{p\left(y_{k} \mid x_{k}\right) p\left(x_{k} \mid D_{k-1}\right)}{\int p\left(y_{k} \mid x_{k}\right) p\left(x_{k} \mid D_{k-1}\right) \mathrm{d} x_{k}},
$$

where the conditional PDF of $y_{k}$ given $x_{k}, p\left(y_{k} \mid x_{k}\right)$, is defined by the measurement model and the known statistics of $v_{k}$

$$
p\left(y_{k} \mid x_{k}\right)=\int \delta\left(y_{k}-h\left(x_{k}\right)-v_{k}\right) p_{v}\left(v_{k}\right) \mathrm{d} v_{k}=p_{v}\left(y_{k}-h\left(x_{k}\right)\right) .
$$

Here $\delta(\cdot)$ is the Dirac function. The delta function arises because if $x_{k}$ and $v_{k}$ are known, then $y_{k}$ is obtained from a purely deterministic relationship (Eq. (13)).

\subsection{Bootstrap filter}

For non-linear problems, analytic solutions to Eqs. (15) and (16) are difficult to be gotten and here the Monte-Carlo method is used to circumvent these difficulties. 
The bootstrap filter is a recursive algorithm to estimate the posterior $p\left(x_{k} \mid D_{k}\right)$ from a set of samples [8]. Suppose we have a set of random samples $\left\{x_{k-1}(i): i=1, \ldots, m\right\}$ from the PDF $p\left(x_{k-1} \mid D_{k-1}\right)$. The bootstrap filter is an algorithm for propagating and updating these samples through the Bayesian recursion to obtain a set of values $\left\{x_{k}(i): i=1, \ldots, m\right\}$, which are approximately distributed as $p\left(x_{k} \mid D_{k}\right)$. The filter procedure is as follows:

(1) Prediction: Each sample from PDF $p\left(x_{k-1} \mid D_{k-1}\right)$ is passed through the system model to obtain samples from the prior at time step $k$ :

$$
x_{k}^{*}(i)=f\left(x_{k-1}(i), w_{k}(i)\right)
$$

where $w_{k}(i)$ is a sample drawn from the PDF of the system noise $p_{w}\left(w_{k}\right)$.

(2) Update: On receipt of the measurement $y_{k}$, evaluate the likelihood of each prior sample and obtain the normalised weight for each sample

$$
q_{i}=\frac{p\left(y_{k} \mid x_{k}^{*}(i)\right)}{\sum_{j=1}^{m} p\left(y_{k} \mid x_{k}^{*}(j)\right)} .
$$

Thus define a discrete distribution over $\left\{x_{k}^{*}(i): i=1, \ldots, m\right\}$, with probability mass $q_{i}$ associated with element $i$. Now resample $m$ times from the discrete distribution to generate samples $\left\{x_{k}(i): i=1, \ldots, m\right\}$, so that for any $j, \operatorname{Pr}\left\{x_{k}(j)=x_{k}^{*}(i)\right\}=q_{i}$. It can be contended that the samples $x_{k}(i)$ are approximately distributed as the required PDF $p\left(x_{k} \mid D_{k}\right)$.

The above steps of prediction and update form a single iteration of the recursive algorithm. Repeat this procedure, we can get $\left\{x_{k}(i): i=1, \ldots, m\right\}$ at every time steps recursively. To initiate the algorithm, $m$ samples $x_{1}^{*}(i)$ are drawn from the known initial PDF $p\left(x_{1} \mid D_{0}\right) \equiv p\left(x_{1}\right)$.

\section{Identification of parameters}

\subsection{Basic philosophy}

Regarding the unknown parameters as state variables, one can define an augmenting state vector $X$ as

$$
\begin{aligned}
X^{T} & =\left\{x, \dot{x}, z, a, b, A, \beta, \gamma, n, \delta_{s}, \sigma\right\} \\
& =\left\{x_{1}, x_{2}, x_{3}, \ldots, x_{10}, x_{11}\right\} .
\end{aligned}
$$

Eqs. (7)-(10) can then be rewritten in the form of non-linear state equations

$$
\dot{X}=f(X, t),
$$


where

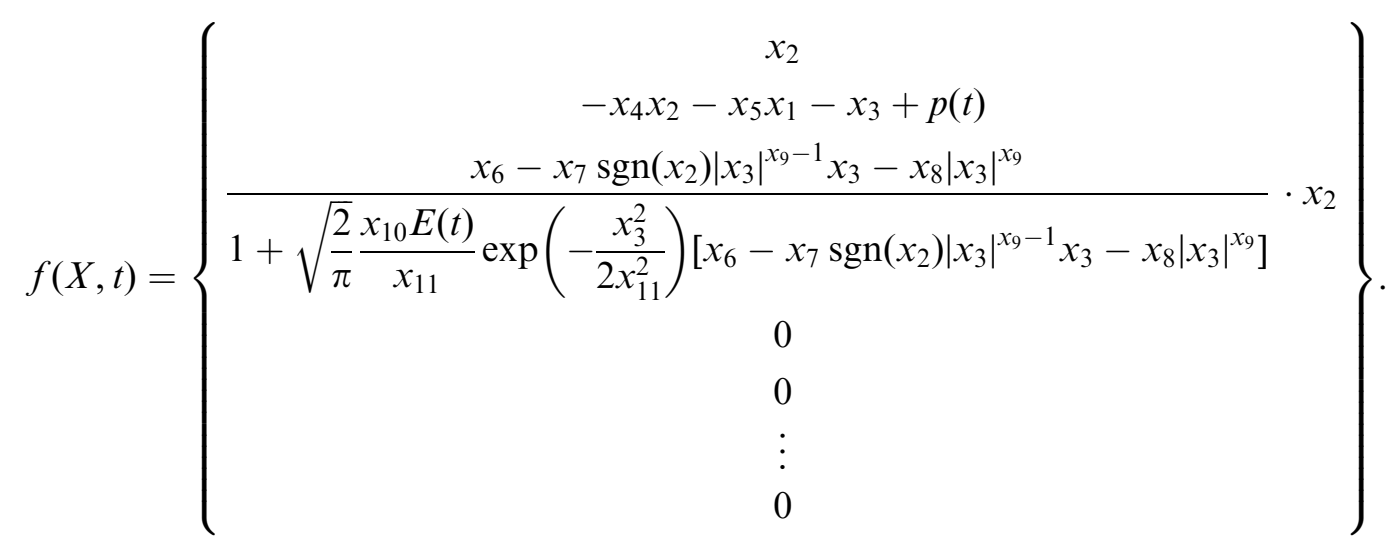

The observation equation here is expressed as

$$
Y=C X+V
$$

where $C$ is the observation matrix and $V$ is the observation noise vector.

Utilising the bootstrap filtering technique in Eqs. (20) and (21), the state vector $X(t)$ can be estimated from the input $p(t)$ and the observed output $\{Y\}_{i}$. Hence, the unknown parameters are estimated simultaneously.

\subsection{Choice of initial estimates}

To start the identification algorithm good initial estimates of parameters are needed. Here a method to decide the initial estimates of parameters of this model is suggested. Noting the fact that $n$ is a positive real number, one can choose a suitable positive value $n_{0}$ as an initial estimate of $n$ at first. The parameters $\delta_{s}$ and $\sigma$ in this model reflect the degree of pinching and the sharpness of loop as shown in Fig. 3. It is not difficult for one to get a suitable initial value of $\delta_{s}$ and $\sigma$ according to the degree of pinching and the sharpness of loops as well as the maximum

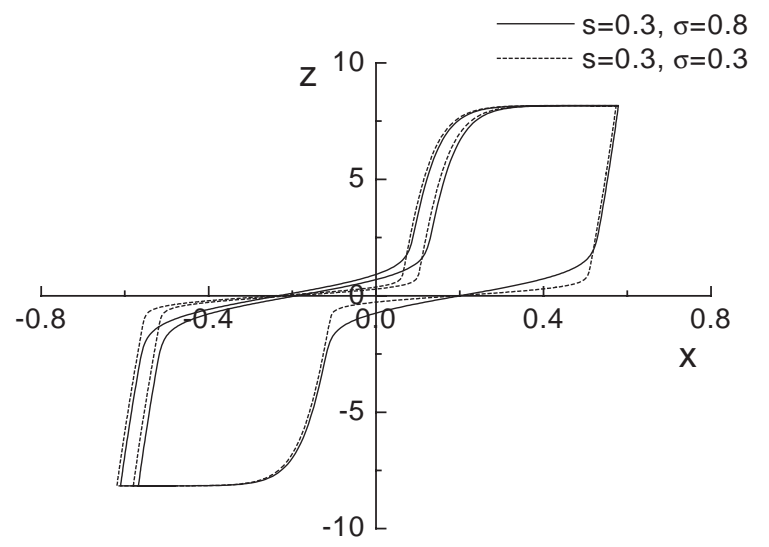

Fig. 3. Pinching behaviour of model under different $\sigma$. 
displacement and the observed dissipated energy of system. Here the corresponding system displacement $x(t)$ of $m$ can be gotten by direct measurement or through integration of $\ddot{x}(t)$.

For the choice of initial estimates of $a$ and $b$, an effective method was obtained in practice by fitting a linear model to the data as follows [15]:

$$
\begin{aligned}
& \int_{0}^{T} \ddot{x}(t) x(t) \mathrm{d} t+\hat{a}_{e q} \int_{0}^{T} \dot{x}(t) x(t) \mathrm{d} t+\hat{b}_{e q} \int_{0}^{T} x^{2}(t) \mathrm{d} t=\int_{0}^{T} p(t) x(t) \mathrm{d} t, \\
& \int_{0}^{T} \ddot{x}(t) \dot{x}(t) \mathrm{d} t+\hat{a}_{e q} \int_{0}^{T} \dot{x}^{2}(t) \mathrm{d} t+\hat{b}_{e q} \int_{0}^{T} x(t) \dot{x}(t) \mathrm{d} t=\int_{0}^{T} p(t) \dot{x}(t) \mathrm{d} t .
\end{aligned}
$$

Here $\ddot{x}(t), \dot{x}(t)$ and $x(t)$ are replaced, respectively, by $\hat{x}(t), \hat{\dot{x}}(t)$ and $\hat{x}(t)$ defined by

$$
\hat{\dot{x}}(t)=\int_{0}^{t} \hat{\ddot{x}}(\tau) \mathrm{d} \tau, \quad \hat{x}(t)=\int_{0}^{t} \hat{\dot{x}}(\tau) \mathrm{d} \tau
$$

and $T$ is the sample length of the selected initial motion records. One can get suitable initial estimates of $a$ and $b$ according to $\hat{a}_{e q}$ and $\hat{b}_{e q}$.

If initial estimates of $a, b, \delta_{s}, n, \sigma$, say $a_{0}, b_{0}, \delta_{s 0}, n_{0}$ and $\sigma_{0}$, are available, a method to estimate the initial values of $A, \beta$ and $\gamma$ is suggested here.

According to the Eqs. (8)-(11) and the assumption of $\operatorname{sgn}\left(\dot{x}_{1}\right)=\operatorname{sgn}(\dot{x})$ [5], an equation about $z$ is yielded as below

$$
\dot{z}=A\left(\dot{x}-\dot{x}_{2}\right)-\beta \operatorname{sgn}(\dot{x})\left(\dot{x}-\dot{x}_{2}\right) z|z|^{n-1}-\gamma\left(\dot{x}-\dot{x}_{2}\right)|z|^{n} .
$$

Here $\operatorname{sgn}(\cdot)$ is the signum function and $\dot{x}_{2}(t)$ is defined by Eq. (9). The initial estimates of $A, \beta$ and $\gamma$ can be gotten by solving the equations from Eq. (23) as follows:

$$
\begin{aligned}
& \overline{x \dot{z}}=\overline{A x\left(\dot{x}-\dot{x}_{2}\right)}-\overline{\beta x \operatorname{sgn}(\dot{x})\left(\dot{x}-\dot{x}_{2}\right) z|z|^{n-1}}-\overline{\gamma x\left(\dot{x}-\dot{x}_{2}\right)|z|^{n}}, \\
& \overline{\dot{x} \dot{z}}=\overline{A \dot{x}\left(\dot{x}-\dot{x}_{2}\right)}-\overline{\beta \dot{x} \operatorname{sgn}(\dot{x})\left(\dot{x}-\dot{x}_{2}\right) z|z|^{n-1}}-\overline{\gamma \dot{x}\left(\dot{x}-\dot{x}_{2}\right)|z|^{n}}, \\
& \overline{\ddot{x} \dot{z}}=\overline{A \ddot{x}\left(\dot{x}-\dot{x}_{2}\right)}-\overline{\beta \ddot{x} \operatorname{sgn}(\dot{x})\left(\dot{x}-\dot{x}_{2}\right) z|z|^{n-1}}-\overline{\gamma \ddot{x}\left(\dot{x}-\dot{x}_{2}\right)|z|^{n}} .
\end{aligned}
$$

The overbars - in equations denote the time averages, for example, $\bar{x}=(1 / T) \int_{0}^{T} x(t) \mathrm{d} t$. And $z(t), \dot{z}(t), \ddot{x}(t), \dot{x}(t), x(t)$ in Eq. (17) are replaced by $\hat{z}(t), \hat{\dot{z}}(t), \hat{\ddot{x}}(t), \hat{\dot{x}}(t)$ and $\hat{x}(t)$ respectively, here $\hat{z}(t)$ and $\hat{\dot{z}}(t)$ are defined as

$$
\begin{aligned}
& \hat{z}(t)=p(t)-\hat{\ddot{x}}(t)-a_{0} \hat{\dot{x}}(t)-b_{0} \hat{x}(t), \\
& \hat{\dot{z}}(t)=\frac{\hat{z}(t+\Delta t)-\hat{z}(t-\Delta t)}{2 \Delta t},
\end{aligned}
$$

in which $\Delta t$ is the sampling interval and $\hat{\dot{x}}(t), \hat{x}(t)$ are defined as the same as Eqs. $(15) . \dot{x}_{2}(t)$ is replaced by $\hat{\dot{x}}_{2}(t)$ defined by

$$
\hat{\dot{x}}_{2}=\sqrt{\frac{2}{\pi}} \frac{\delta_{s 0} \hat{E}(t)}{\sigma_{0}} \exp \left[-\frac{\hat{z}^{2}}{2 \sigma_{0}^{2}}\right] \hat{\dot{z}} .
$$


Here $\hat{E}(t)$ is computed by

$$
\hat{E}(t)=\int_{0}^{t} \hat{z}(t) \mathrm{d} x=\int_{0}^{t} \hat{z}(t) \hat{\dot{x}}(t) \mathrm{d} t .
$$

\section{Numerical example and discussions}

In order to validate the proposed identification method, a numerical simulation to a singledegree-of-freedom hysteretic element have been performed. The element was subjected to an excitation and its responses recorded. These response records were then used to identify the parameter values of this hysteretic element. The model parameters were selected to have the following values:

$$
a=0.5, \quad b=30, \quad A=20, \quad \beta=5, \quad \gamma=5, \quad n=2, \quad \delta_{s}=0.06, \quad \sigma=0.05
$$

and the El Centro earthquake (NS, 1940) was used as the input excitation as shown in Fig. 4. Eqs. (7)-(11) with these parameters and excitation were solved numerically by using the

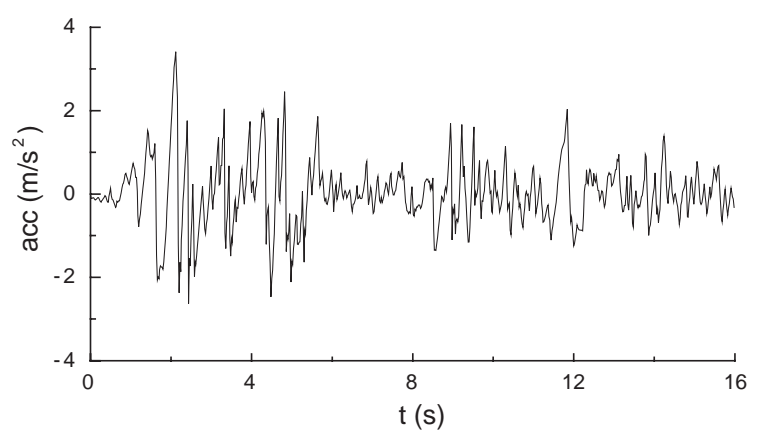

Fig. 4. El Centro earthquake (NS, 1940).

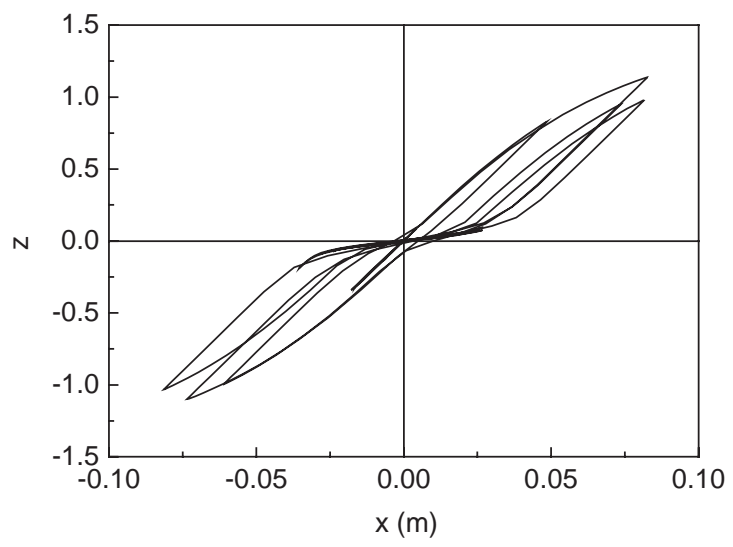

Fig. 5. Hysteresis loops. 
fourth-order Runge-Kutta method, the interval of time used here was $\Delta t=0.02 \mathrm{~s}$. The hysteresis loops are shown in Fig. 5.

In this simulation, assume the experimental measurement is the displacement, which is the solved $x(t)$ (as shown in Fig. 6) contaminated by noise $v_{k}$. The observation equation (21) is then given as follows:

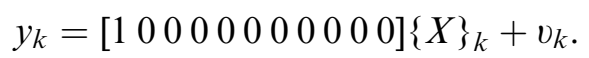

Here the noise $v_{k}$ is assumed zero mean random process, which has the Student's $t$ distribution with four degrees of freedom scaled according to the standard deviation of the principal noise component. This distribution has heavier tails than the Gaussian and so models occasional, exceptionally large measurement errors, i.e., it accommodates the outliers [18]. Thus, the PDF of

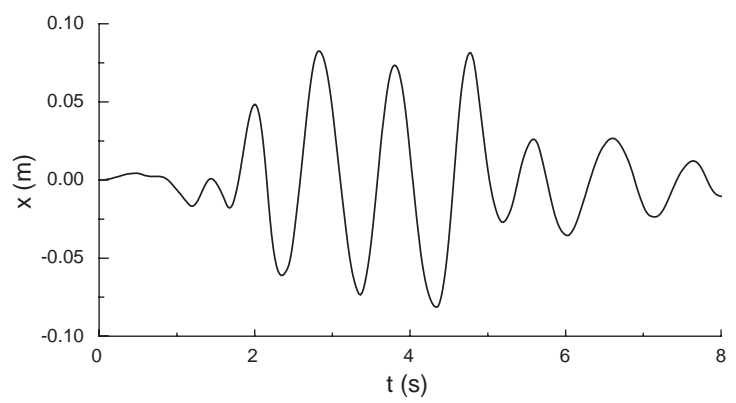

Fig. 6. Displacement response.

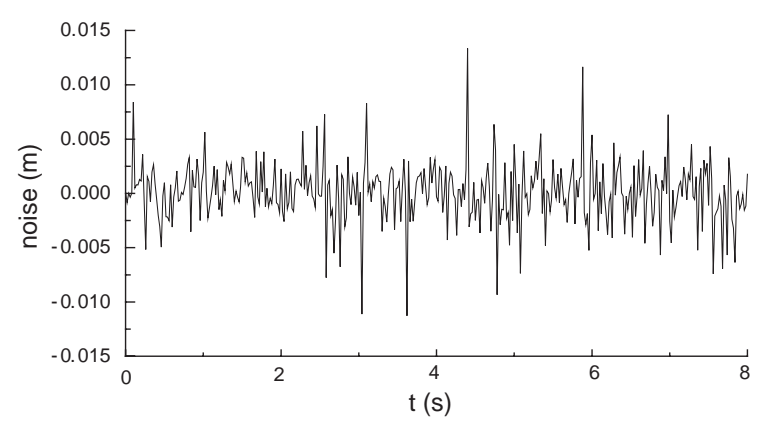

Fig. 7. A noise sample with $t$ distribution.

Table 1

Initial conditions

\begin{tabular}{llllllllllll}
\hline Variable & $x$ & $\dot{x}$ & $z$ & $a$ & $b$ & $A$ & $\beta$ & $\gamma$ & $n$ & $\delta_{s}$ & $\sigma$ \\
\hline $\bar{x}\left(t_{0} \mid t_{0}\right)$ & 0 & 0 & 0 & 0.28 & 35 & 30 & 6.5 & 6.8 & 1.8 & 0.03 & 0.08 \\
$P\left(t_{0} \mid t_{0}\right)$ & 0.001 & 0.001 & 0.001 & 0.1 & 80 & 150 & 20 & 20 & 0.01 & 0.01 & 0.01 \\
\hline
\end{tabular}


the measurement error is modelled as

$$
p(v) \propto\left(n+v^{2} / s^{2}\right)^{-(n+1) / 2},
$$

where degree of freedom $n=4$ and scale $s=0.002$. Note that since the update weights are normalised (Eq. (18)), the measurement error PDF need only be specified to proportionality. Fig. 7 is a noise sample simulated from this distribution.

The initial conditions for this analysis were estimated according to the method proposed in this paper and given in Table 1. The distributions of all the initial estimations are assumed to be Gaussian and the total number of sample realisations $m=1000$. It should be noted that the assumption of Gaussian distribution here is not a requirement.

Table 2

Identified parameters

\begin{tabular}{lllllllll}
\hline Parameter & $a$ & $b$ & $A$ & $\beta$ & $\gamma$ & $n$ & $\delta_{s}$ & $\sigma$ \\
\hline Exact value & 0.5 & 30 & 20 & 5 & 5 & 2 & 0.06 & 0.05 \\
Identified value & 0.4684 & 31.3811 & 17.4806 & 4.1097 & 5.8134 & 1.8833 & 0.0454 & 0.0545 \\
\hline
\end{tabular}

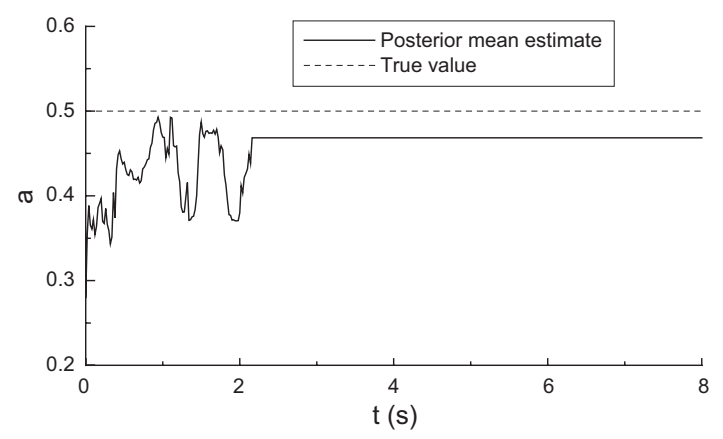

Fig. 8. Mean of parameter $a$.

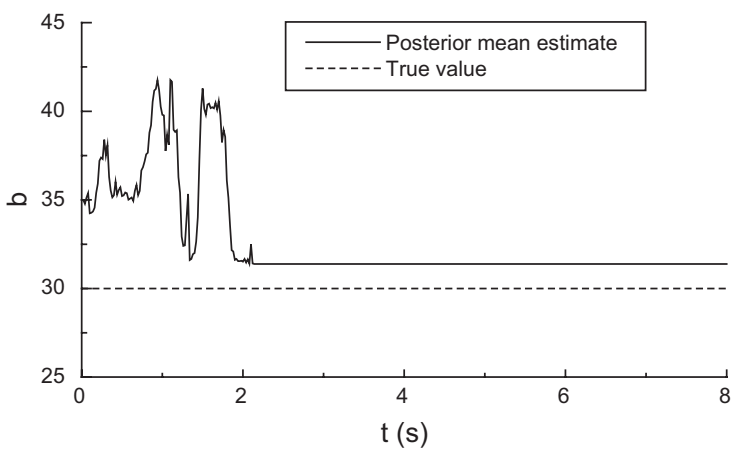

Fig. 9. Mean of parameter $b$. 


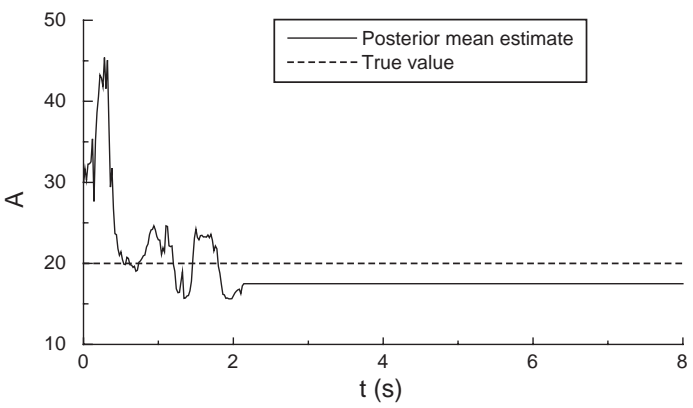

Fig. 10. Mean of parameter $A$.

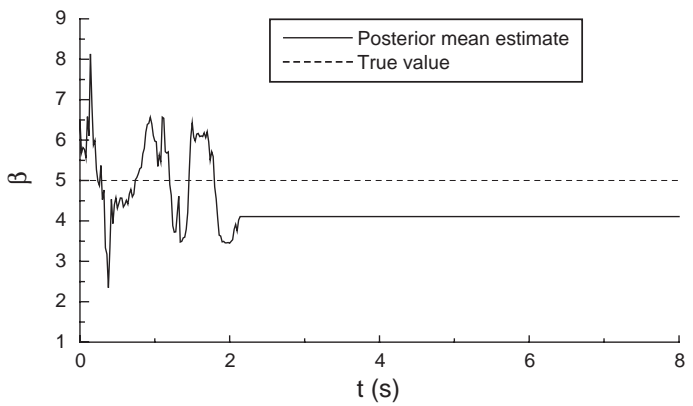

Fig. 11. Mean of parameter $\beta$.

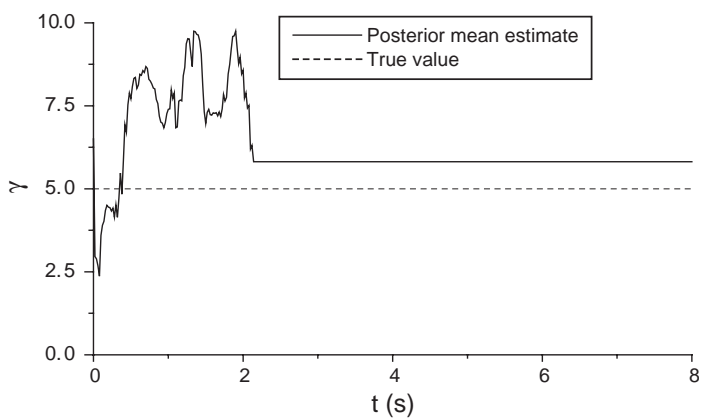

Fig. 12. Mean of parameter $\gamma$.

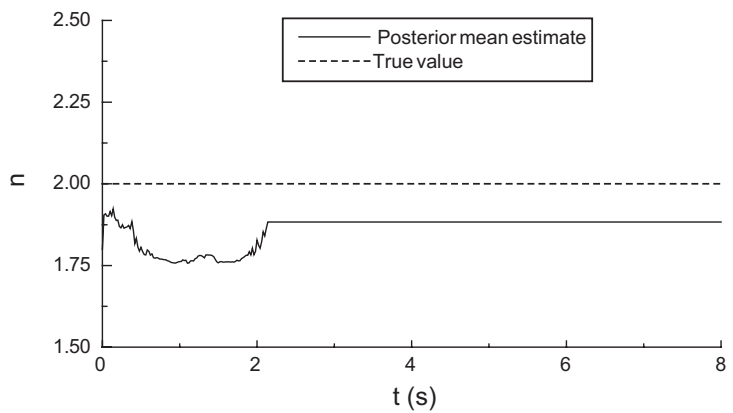

Fig. 13. Mean of parameter $n$. 


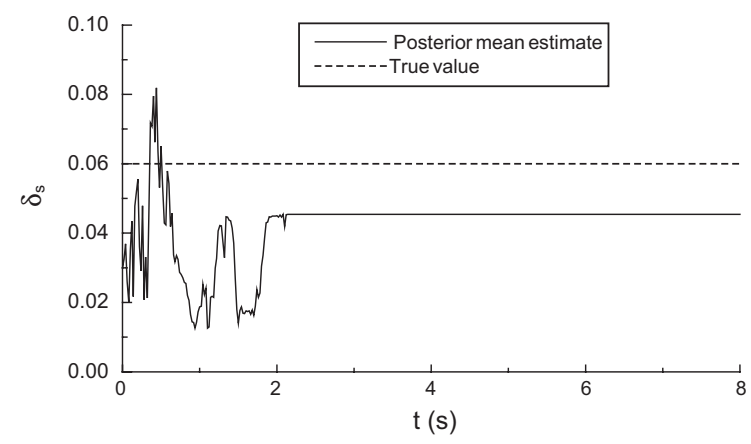

Fig. 14. Mean of parameter $\delta_{s}$.

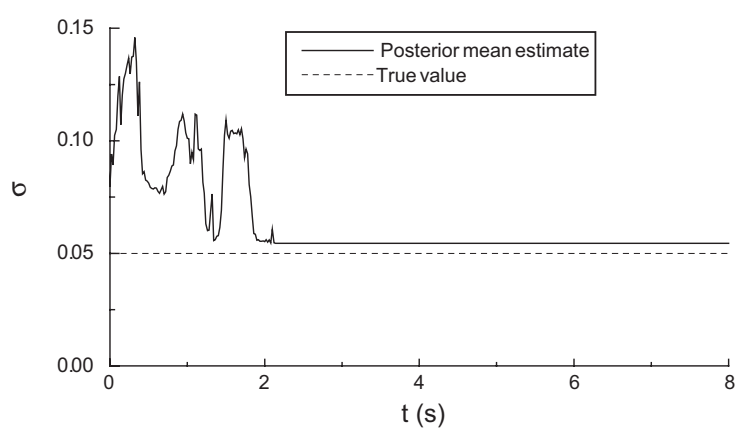

Fig. 15. Mean of parameter $\sigma$.

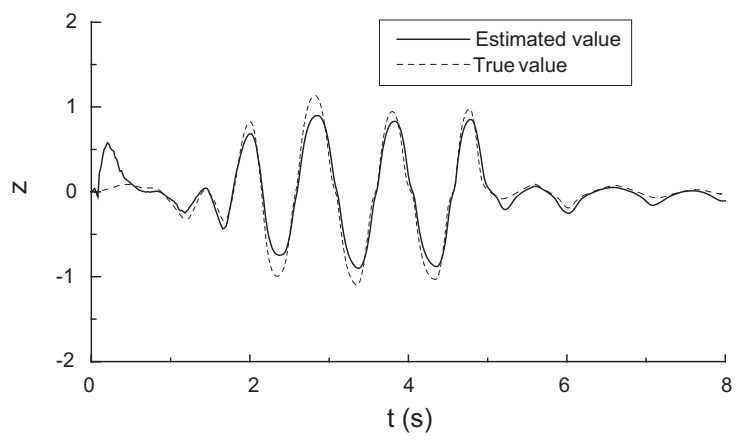

Fig. 16. Comparison of actual and predicted hysteretic restoring force $z$.

The parameters are estimated by the identification method proposed in this paper, and the estimated results are given in Table 2. The distribution of process noise $p_{w}\left(w_{k}\right)$ is assumed normal with zero mean and standard deviation 0.3 in this simulation. Figs. $8-15$ show the time histories of the mean of estimated parameters. It can be seen that with the improvement of time the parameters are convergence to good estimated values, although there are great divergence in initial. 


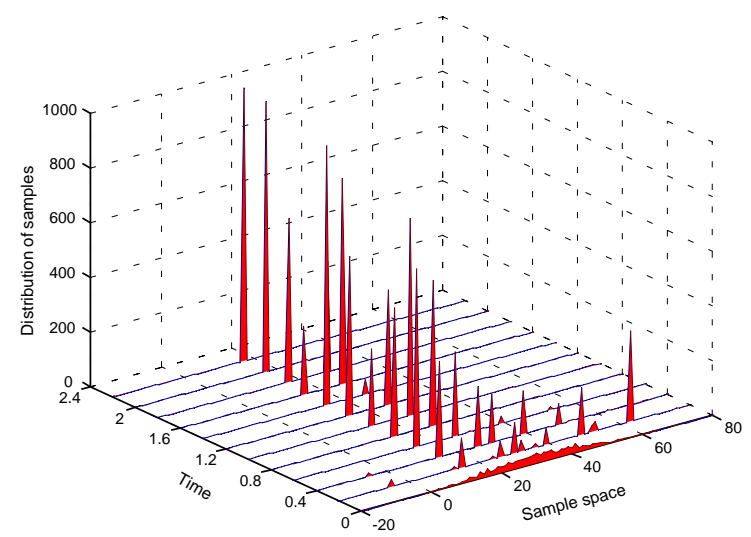

Fig. 17. Sample distributions of parameter $A$.

Fig. 16 compares the actual hysteretic restoring force with the predicted $z$. The agreement is very good. Fig. 17 shows the sample distributions of parameter $A$ at different time step. It can be seen clearly that the sample distributions of parameter $A$ are not Gaussian in time direction even if the distribution of initial estimation is Gaussian.

The simulations with the displacement $x(t)$ corrupted by different level of noise were also carried out and the proposed method can produce effective identification results although the estimates for the higher level of noise were poor as compared to those with lower noise level and the choice of initial estimates are more crucial.

\section{Conclusion}

Using bootstrap filtering technique, a method of estimating the parameters of non-linear hysteretic system with slip on the basis of input-output data has been suggested. It is shown that the proposed approach can yield reliable estimates of system even in the case of very severe material non-linearity. And it is not restricted by model assumption of Gaussian noise. The method is ideally suited for the identification of non-linear hysteretic systems with slip typically encountered in the applied mechanics field.

For the hysteretic slip model presented, the parameters except $\delta_{s}$ are considered to be constant, i.e., these parameters do not vary with the progress of response. But in practice, the parameters may be varied as a function of the response history to introduce system deterioration. In general the parameters are also supposed as a function of the system energy dissipation $E(t)$. The identification method presented here is also suitable for this case. The treatments on these parameters are as the same as done on the parameter $\delta_{s}$.

\section{References}

[1] Y. Suzuki, R. Minai, Application of stochastic differential equations to seismic reliability analysis of hysteretic structures, Probabilistic Engineering Mechanics 3 (1988) 43-52. 
[2] R. Bouc, Forced vibration of mechanical system with hysteresis, Proceedings of the Fourth Conference on Nonlinear Oscillations, Prague, 1967, p. 315.

[3] Y.K. Wen, Method for random vibration of hysteretic systems, Journal of Engineering Mechanics 102 (1976) 249-263.

[4] T.T. Baber, Y.K. Wen, Random vibration of hysteretic degrading systems, Journal of Engineering Mechanics 107 (1981) 1069-1089.

[5] T.T. Baber, M.N. Noori, Random vibration of degrading pinching systems, Journal of Engineering Mechanics 111 (1985) 1010-1026.

[6] M. Hoshiya, E. Saito, Structural identification by extended Kalman filter, Journal of Engineering Mechanics 110 (1984) $1757-1770$.

[7] C.-B. Yun, M. Shinozuka, Identification of nonlinear structural dynamic systems, Journal of Structural Mechanics 8 (1980) 187-203.

[8] N.J. Gordon, D.J. Salmond, A.F.M. Smith, Novel approach to nonlinear/non-Gaussian Bayesian state estimation, IEE Proceedings-F 140 (1993) 107-113.

[9] G. Kitagawa, Monte Carlo filter and smoother for non-Gaussian non-linear state space models, Journal of Computational and Graphical Statistics 5 (1996) 1-25.

[10] M. Hoshiya, O. Maruyama, State estimation of conditional non-Gaussian random fields by BF/MCF, Proceedings of U.S.-Japan Cooperative Research on Urban Earthquake Disaster Mitigation: U.S.-Japan Joint Workshop and Third Grantees Meeting, University of Washington, Seattle, Washington, USA, 2001, pp. 226-236.

[11] M. Hoshiya, I. Yoshida, Identification of conditional stochastic Gaussian field, Journal of Engineering Mechanics 122 (1996) 101-108.

[12] M. Hoshiya, S. Noda, H. Inada, Estimation of conditional non-Gaussian translation stochastic fields, Journal of Engineering Mechanics 124 (1998) 435-445.

[13] T. Sato, K. Kaji, Adaptive Monte Carlo filter and structural identification, Proceedings of International Conference on Monte Carlo Simulation, Monaco, June 18-21, 2000, pp. 441-447.

[14] R.H. Sues, S.T. Mau, Y.K. Wen, Systems identification of degrading hysteretic restoring forces, Journal of Engineering Mechanics 114 (1988) 833-846.

[15] M. Yar, J.K. Hammond, Parameter estimation for hysteretic systems, Journal of Sound and Vibration 117 (1987) $161-172$.

[16] A.G. Chassiakos, S.F. Masri, A.W. Smyth, T.K. Caughey, On-line identification of hysteretic systems, Journal of Applied Mechanics 65 (1998) 194-203.

[17] J.W. Lin, R. Betti, A.W. Smyth, R.W. Longman, On-line identification of non-linear hysteretic structural systems using a variable trace approach, Earthquake Engineering and Structural Dynamics 30 (2001) 1279-1303.

[18] N. Gordon, D. Salmond, C. Ewing, Bayesian state estimation for tracking and guidance using the bootstrap filter, Journal of Guidance, Control and Dynamics 18 (1995) 1434-1443. 